\title{
An Evaluation Exercise for Word Alignment
}

\author{
Rada Mihalcea \\ Department of Computer Science \\ University of North Texas \\ Denton, TX 76203 \\ rada@cs.unt.edu
}

\author{
Ted Pedersen \\ Department of Computer Science \\ University of Minnesota \\ Duluth, MN 55812 \\ tpederse@umn.edu
}

\begin{abstract}
This paper presents the task definition, resources, participating systems, and comparative results for the shared task on word alignment, which was organized as part of the HLT/NAACL 2003 Workshop on Building and Using Parallel Texts. The shared task included Romanian-English and English-French sub-tasks, and drew the participation of seven teams from around the world.
\end{abstract}

\section{Defining a Word Alignment Shared Task}

The task of word alignment consists of finding correspondences between words and phrases in parallel texts. Assuming a sentence aligned bilingual corpus in languages $\mathrm{L} 1$ and L2, the task of a word alignment system is to indicate which word token in the corpus of language L1 corresponds to which word token in the corpus of language L2.

As part of the HLT/NAACL 2003 workshop on "Building and Using Parallel Texts: Data Driven Machine Translation and Beyond", we organized a shared task on word alignment, where participating teams were provided with training and test data, consisting of sentence aligned parallel texts, and were asked to provide automatically derived word alignments for all the words in the test set. Data for two language pairs were provided: (1) EnglishFrench, representing languages with rich resources (20 million word parallel texts), and (2) Romanian-English, representing languages with scarce resources $(1$ million word parallel texts). Similar with the Machine Translation evaluation exercise organized by NIST $^{1}$, two subtasks were defined, with teams being encouraged to participate in both subtasks.

\footnotetext{
${ }^{1}$ http://www.nist.gov/speech/tests/mt/
}

1. Limited resources, where systems are allowed to use only the resources provided.

2. Unlimited resources, where systems are allowed to use any resources in addition to those provided. Such resources had to be explicitly mentioned in the system description.

Test data were released one week prior to the deadline for result submissions. Participating teams were asked to produce word alignments, following a common format as specified below, and submit their output by a certain deadline. Results were returned to each team within three days of submission.

\subsection{Word Alignment Output Format}

The word alignment result files had to include one line for each word-to-word alignment. Additionally, lines in the result files had to follow the format specified in Fig.1.

While the $S \mid P$ and confidence fields overlap in their meaning, the intent of having both fields available is to enable participating teams to draw their own line on what they consider to be a Sure or Probable alignment. Both these fields were optional, with some standard values assigned by default.

\subsubsection{A Running Word Alignment Example}

Consider the following two aligned sentences: [English] $<$ s snum $=18>$ They had gone $.</ s>$ [French] $<s$ snum $=18>$ Ils etaient alles . $</ s>$

A correct word alignment for this sentence is

1811

1822

1833

1844

stating that: all the word alignments pertain to sentence 18, the English token 1 They aligns with the French token $1 \mathrm{Ils}$, the English token $2 \mathrm{had}$, aligns with the French token 2 etaient, and so on. Note that punctuation is also 


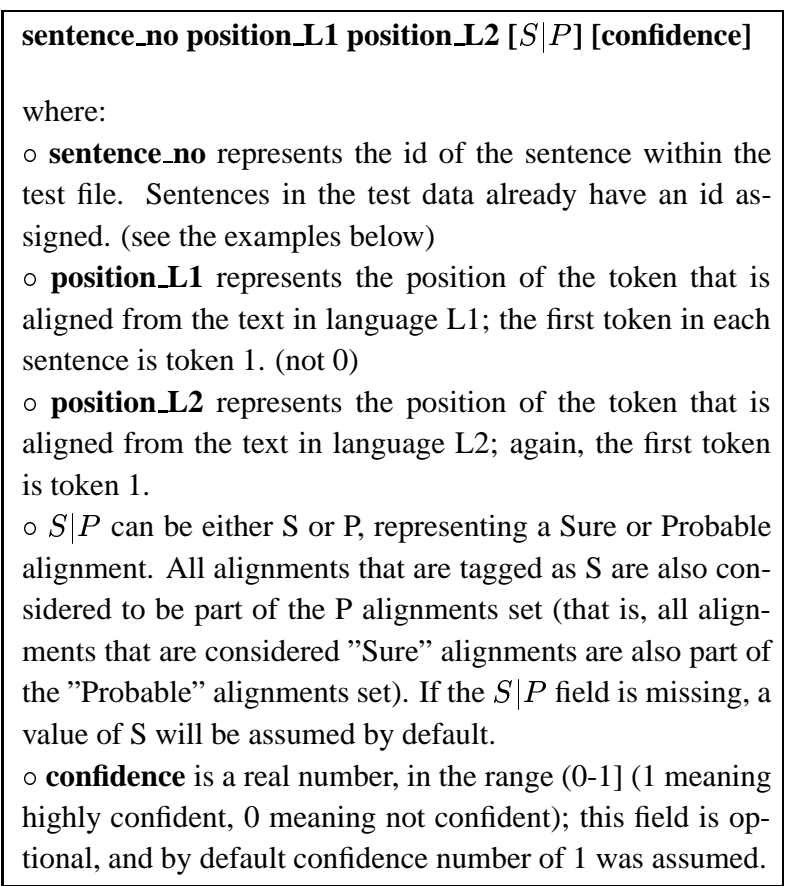

Figure 1: Word Alignment file format

aligned (English token 4 aligned with French token 4), and counts towards the final evaluation figures.

Alternatively, systems could also provide an $S \mid P$ marker and/or a confidence score, as shown in the following example:

18111

$1822 \mathrm{P} 0.7$

$1833 \mathrm{~S}$

$1844 \mathrm{~S} 1$

with missing $S \mid P$ fields considered by default to be $\mathrm{S}$, and missing confidence scores considered by default 1 .

\subsection{Annotation Guide for Word Alignments}

The annotation guide and illustrative word alignment examples were mostly drawn from the Blinker Annotation Project. Please refer to (Melamed, 1999, pp. 169-182) for additional details.

1. All items separated by a white space are considered to be a word (or token), and therefore have to be aligned. (punctuation included)

2. Omissions in translation use the NULL token, i.e. token with id 0 . For instance, in the examples below:

[English]: $<s$ snum $=18>$ And he said, appoint me thy wages, and I will give it . $</ s>$

[French]: $<s$ snum $=18>$ fixe moi ton salaire, et je te le donnerai . $\langle/ s\rangle$ and he said from the English sentence has no corresponding translation in French, and therefore all these words are aligned with the token id 0 .

‥

1810

1820

1830

1840

$\cdots$

3. Phrasal correspondences produce multiple word-toword alignments. For instance, in the examples below:

English: $<s$ snum $=18>$ cultiver la terre $</ s>$

French: $<s$ snum $=18>$ to be a husbandman $</ s>$

since the words do not correspond one to one, and yet the two phrases mean the same thing in the given context, the phrases should be linked as wholes, by linking each word in one to each word in another. For the example above, this translates into 12 wordto-word alignments:

\begin{tabular}{|c|c|}
\hline 1811 & 1812 \\
\hline 1813 & 1814 \\
\hline 1821 & 1822 \\
\hline 1823 & 1824 \\
\hline 1831 & 1832 \\
\hline 1833 & 1834 \\
\hline
\end{tabular}

\section{Resources}

The shared task included two different language pairs: the alignment of words in English-French parallel texts, and in Romanian-English parallel texts. For each language pair, training data were provided to participants. Systems relying only on these resources were considered part of the Limited Resources subtask. Systems making use of any additional resources (e.g. bilingual dictionaries, additional parallel corpora, and others) were classified under the Unlimited Resources category.

\subsection{Training Data}

Two sets of training data were made available.

1. A set of Romanian-English parallel texts, consisting of about 1 million Romanian words, and about the same number of English words. These data consisted of:

- Parallel texts collected from the Web using a semi-supervised approach. The URLs format for pages containing potential parallel translations were manually identified (mainly from the archives of Romanian newspapers). Next, texts were automatically downloaded and sentence aligned. A manual verification of the 
alignment was also performed. These data collection process resulted in a corpus of about 850,000 Romanian words, and about 900,000 English words.

- Orwell's 1984, aligned within the MULTEXTEAST project (Erjavec et al., 1997), with about 130,000 Romanian words, and a similar number of English words.

- The Romanian Constitution, for about 13,000 Romanian words and 13,000 English words.

2. A set of English-French parallel texts, consisting of about 20 million English words, and about the same number of French words. This is a subset of the Canadian Hansards, processed and sentence aligned by Ulrich Germann at ISI (Germann, 2001).

All data were pre-tokenized. For English and French, we used a version of the tokenizers provided within the EGYPT Toolkit ${ }^{2}$. For Romanian, we used our own tokenizer. Identical tokenization procedures were used for training, trial, and test data.

\subsection{Trial Data}

Two sets of trial data were made available at the same time training data became available. Trial sets consisted of sentence aligned texts, provided together with manually determined word alignments. The main purpose of these data was to enable participants to better understand the format required for the word alignment result files. Trial sets consisted of 37 English-French, and 17 Romanian-English aligned sentences.

\subsection{Test Data}

A total of 447 English-French aligned sentences (Och and Ney, 2000), and 248 Romanian-English aligned sentences were released one week prior to the deadline. Participants were required to run their word alignment systems on these two sets, and submit word alignments. Teams were allowed to submit an unlimited number of results sets for each language pair.

\subsubsection{Gold Standard Word Aligned Data}

The gold standard for the two language pair alignments were produced using slightly different alignment procedures, which allowed us to study different schemes for producing gold standards for word aligned data.

For English-French, annotators where instructed to assign a Sure or Probable tag to each word alignment they produced. The intersection of the Sure alignments produced by the two annotators led to the final Sure aligned set, while the reunion of the Probable alignments led to the final Probable aligned set. The Sure alignment set is

\footnotetext{
${ }^{2}$ http://www.clsp.jhu.edu/ws99/projects/mt/toolkit/
}

guaranteed to be a subset of the Probable alignment set. The annotators did not produce any NULL alignments. Instead, we assigned NULL alignments as a default backup mechanism, which forced each word to belong to at least one alignment. The English-French aligned data were produced by Franz Och and Hermann Ney (Och and Ney, 2000).

For Romanian-English, annotators were instructed to assign an alignment to all words, with specific instructions as to when to assign a NULL alignment. Annotators were not asked to assign a Sure or Probable label. Instead, we had an arbitration phase, where a third annotator judged the cases where the first two annotators disagreed. Since an inter-annotator agreement was reached for all word alignments, the final resulting alignments were considered to be Sure alignments.

\section{Evaluation Measures}

Evaluations were performed with respect to four different measures. Three of them - precision, recall, and Fmeasure - represent traditional measures in Information Retrieval, and were also frequently used in previous word alignment literature. The fourth measure was originally introduced by (Och and Ney, 2000), and proposes the notion of quality of word alignment.

Given an alignment $\mathcal{A}$, and a gold standard alignment $\mathcal{G}$, each such alignment set eventually consisting of two sets $\mathcal{A}_{\mathcal{S}}, \mathcal{A}_{\mathcal{P}}$, and $\mathcal{G}_{\mathcal{S}}, \mathcal{G}_{\mathcal{P}}$ corresponding to Sure and Probable alignments, the following measures are defined (where $T$ is the alignment type, and can be set to either $\mathrm{S}$ or $\mathrm{P})$.

$$
\begin{gathered}
P_{T}=\frac{\left|A_{T} \cap G_{T}\right|}{\left|A_{T}\right|} \\
R_{T}=\frac{\left|A_{T} \cap G_{T}\right|}{\left|G_{T}\right|} \\
F_{T}=\frac{2 P_{T} R_{T}}{P_{T}+R_{T}} \\
A E R=1-\frac{\left|A_{P} \cap G_{S}\right|+\left|A_{P} \cap G_{P}\right|}{\left|A_{P}\right|+\left|G_{S}\right|}
\end{gathered}
$$

Each word alignment submission was evaluated in terms of the above measures. Moreover, we conducted two sets of evaluations for each submission:

- NULL-Align, where each word was enforced to belong to at least one alignment; if a word did not belong to any alignment, a NULL Probable alignment was assigned by default. This set of evaluations pertains to full coverage word alignments.

- NO-NULL-Align, where all NULL alignments were removed from both submission file and gold standard data. 


\begin{tabular}{|l|l|l|}
\hline Team & System name & Description \\
\hline \hline Language Technologies Institute, CMU & BiBr & (Zhao and Vogel, 2003) \\
MITRE Corporation & Fourday & (Henderson, 2003) \\
RALI - Université the Montréal & Ralign & (Simard and Langlais, 2003) \\
Romanian Academy Institute of Artificial Intelligence & RACAI & (Tufiş et al., 2003) \\
University of Alberta & ProAlign & (Lin and Cherry, 2003) \\
University of Minnesota, Duluth & UMD & (Thomson McInnes and Pedersen, 2003) \\
Xerox Research Centre Europe & XRCE & (Dejean et al., 2003) \\
\hline
\end{tabular}

Table 1: Teams participating in the word alignment shared task

We conducted therefore 14 evaluations for each submission file: AER, Sure/Probable Precision, Sure/Probable Recall, and Sure/Probable F-measure, with a different figure determined for NULL-Align and NO-NULL-Align alignments.

\section{Participating Systems}

Seven teams from around the world participated in the word alignment shared task. Table 1 lists the names of the participating systems, the corresponding institutions, and references to papers in this volume that provide detailed descriptions of the systems and additional analysis of their results.

All seven teams participated in the Romanian-English subtask, and five teams participated in the English-French subtask. ${ }^{3}$ There were no restrictions placed on the number of submissions each team could make. This resulted in a total of 27 submissions from the seven teams, where 14 sets of results were submitted for the English-French subtask, and 13 for the Romanian-English subtask. Of the 27 total submissions, there were 17 in the Limited resources subtask, and 10 in the Unlimited resources subtask. Tables 2 and 3 show all of the submissions for each team in the two subtasks, and provide a brief description of their approaches.

While each participating system was unique, there were a few unifying themes.

Four teams had approaches that relied (to varying degrees) on an IBM model of statistical machine translation (Brown et al., 1993). UMD was a straightforward implementation of IBM Model 2, BiBr employed a boosting procedure in deriving an IBM Model 1 lexicon, Ralign used IBM Model 2 as a foundation for their recursive splitting procedure, and XRCE used IBM Model 4 as a base for alignment with lemmatized text and bilingual lexicons.

Two teams made use of syntactic structure in the text to be aligned. ProAlign satisfies constraints derived from a dependency tree parse of the English sentence being

\footnotetext{
${ }^{3}$ The two teams that did not participate in English-French were Fourday and RACAI.
}

aligned. $\mathrm{BiBr}$ also employs syntactic constraints that must be satisfied. However, these come from parallel text that has been shallowly parsed via a method known as bilingual bracketing.

Three teams approached the shared task with baseline or prototype systems. Fourday combines several intuitive baselines via a nearest neighbor classifier, RACAI carries out a greedy alignment based on an automatically extracted dictionary of translations, and UMD's implementation of IBM Model 2 provides an experimental platform for their future work incorporating prior knowledge about cognates. All three of these systems were developed within a short period of time before and during the shared task.

\section{Results and Discussion}

Tables 4 and 5 list the results obtained by participating systems in the Romanian-English task. Similarly, results obtained during the English-French task are listed in Tables 6 and 7 .

For Romanian-English, limited resources, XRCE systems (XRCE.Nolem-56k.RE.2 and XRCE.Trilex.RE.3) seem to lead to the best results. These are systems that are based on GIZA++, with or without additional resources (lemmatizers and lexicons). For unlimited resources, ProAlign.RE.1 has the best performance.

For English-French, Ralign.EF.1 has the best performance for limited resources, while ProAlign.EF.1 has again the largest number of top ranked figures for unlimited resources.

To make a cross-language comparison, we paid particular attention to the evaluation of the Sure alignments, since these were collected in a similar fashion (an agreement had to be achieved between two different annotators). The results obtained for the English-French Sure alignments are significantly higher $(80.54 \%$ best Fmeasure) than those for Romanian-English Sure alignments (71.14\% best F-measure). Similarly, AER for English-French (5.71\% highest error reduction) is clearly better than the AER for Romanian-English (28.86\% highest error reduction). 
This difference in performance between the two data sets is not a surprise. As expected, word alignment, like many other NLP tasks (Banko and Brill, 2001), highly benefits from large amounts of training data. Increased performance is therefore expected when larger training data sets are available.

The only evaluation set where Romanian-English data leads to better performance is the Probable alignments set. We believe however that these figures are not directly comparable, since the English-French Probable alignments were obtained as a reunion of the alignments assigned by two different annotators, while for the Romanian-English Probable set two annotators had to reach an agreement (that is, an intersection of their individual alignment assignments).

Interestingly, in an overall evaluation, the limited resources systems seem to lead to better results than those with unlimited resources. Out of 28 different evaluation figures, 20 top ranked figures are provided by systems with limited resources. This suggests that perhaps using a large number of additional resources does not seem to improve a lot over the case when only parallel texts are employed.

Ranked results for all systems are plotted in Figures 2 and 3. In the graphs, systems are ordered based on their AER scores. System names are preceded by a marker to indicate the system type: L stands for Limited Resources, and U stands for Unlimited Resources.

\section{Conclusion}

A shared task on word alignment was organized as part of the HLT/NAACL 2003 Workshop on Building and Using Parallel Texts. In this paper, we presented the task definition, and resources involved, and shortly described the participating systems. The shared task included Romanian-English and English-French sub-tasks, and drew the participation of seven teams from around the world. Comparative evaluations of results led to interesting insights regarding the impact on performance of (1) various alignment algorithms, (2) large or small amounts of training data, and (3) type of resources available. Data and evaluation software used in this exercise are available online at http://www.cs.unt.edu/ rada/wpt.

\section{Acknowledgments}

There are many people who contributed greatly to making this word alignment evaluation task possible. We are grateful to all the participants in the shared task, for their hard work and involvement in this evaluation exercise. Without them, all these comparative analyses of word alignment techniques would not be possible.

We are very thankful to Franz Och from ISI and Hermann Ney from RWTH Aachen for kindly making their
English-French word aligned data available to the workshop participants; the Hansards made available by Ulrich Germann from ISI constituted invaluable data for the English-French shared task. We would also like to thank the student volunteers from the Department of English, Babes-Bolyai University, Cluj-Napoca, Romania who helped creating the Romanian-English word aligned data.

We are also grateful to all the Program Committee members of the current workshop, for their comments and suggestions, which helped us improve the definition of this shared task. In particular, we would like to thank Dan Melamed for suggesting the two different subtasks (limited and unlimited resources), and Michael Carl and Phil Resnik for initiating interesting discussions regarding phrase-based evaluations.

\section{References}

M. Banko and E. Brill. 2001. Scaling to very very large corpora for natural language disambiguation. In Proceedings of the 39th Annual Meeting of the Association for Computational Lingusitics (ACL-2001), Toulouse, France, July.

P. Brown, S. Della Pietra, V. Della Pietra, and R. Mercer. 1993. The mathematics of statistical machine translation: Parameter estimation. Computational Linguistics, 19(2):263-311.

Herve Dejean, Eric Gaussier, Cyril Goutte, and Kenji Yamada. 2003. Reducing parameter space for word alignment. In HLT-NAACL 2003 Workshop: Building and Using Parallel Texts: Data Driven Machine Translation and Beyond, pages 23-26, Edmonton, Alberta, Canada, May 31. Association for Computational Linguistics.

T. Erjavec, N. Ide, and D. Tufiş. 1997. Encoding and parallel alignment of linguistic corpora in six central and Eastern European languages. In Proceedings of the Joint ACH/ALL Conference, Queen's University, Kingston, Ontario, June.

U. Germann. 2001. Aligned hansards of the 36th parliament of canada. http://www.isi.edu/naturallanguage/download/hansard/.

John C. Henderson. 2003. Word alignment baselines. In HLT-NAACL 2003 Workshop: Building and Using Parallel Texts: Data Driven Machine Translation and Beyond, pages 27-30, Edmonton, Alberta, Canada, May 31. Association for Computational Linguistics.

Dekang Lin and Colin Cherry. 2003. Proalign: Shared task system description. In HLT-NAACL 2003 Workshop: Building and Using Parallel Texts: Data Driven 
Machine Translation and Beyond, pages 11-14, Edmonton, Alberta, Canada, May 31. Association for Computational Linguistics.

D.I. Melamed. 1999. Empirical Methods for Exploiting Parallel Texts. MIT Press.

F. Och and H. Ney. 2000. A comparison of alignment models for statistical machine translation. In Proceedings of the 18th International Conference on Computational Linguistics (COLING-ACL 2000), Saarbrucken, Germany, August.

Michel Simard and Philippe Langlais. 2003. Statistical translation alignment with compositionality constraints. In HLT-NAACL 2003 Workshop: Building and Using Parallel Texts: Data Driven Machine Translation and Beyond, pages 19-22, Edmonton, Alberta, Canada, May 31. Association for Computational Linguistics.

Bridget Thomson McInnes and Ted Pedersen. 2003. The duluth word alignment system. In HLT-NAACL 2003 Workshop: Building and Using Parallel Texts: Data Driven Machine Translation and Beyond, pages 4043, Edmonton, Alberta, Canada, May 31. Association for Computational Linguistics.

Dan Tufiş, Ana-Maria Barbu, and Radu Ion. 2003. Treqal: A word alignment system with limited language resources. In HLT-NAACL 2003 Workshop: Building and Using Parallel Texts: Data Driven Machine Translation and Beyond, pages 36-39, Edmonton, Alberta, Canada, May 31. Association for Computational Linguistics.

D Tufiş. 2002. A cheap and fast way to build useful translation lexicons. In Proceedings of the 19th International Conference on Computational Linguistics, pages 1030-1036, Taipei, August.

Bing Zhao and Stephan Vogel. 2003. Word alignment based on bilingual bracketing. In HLT-NAACL 2003 Workshop: Building and Using Parallel Texts: Data Driven Machine Translation and Beyond, pages 1518, Edmonton, Alberta, Canada, May 31. Association for Computational Linguistics. 


\begin{tabular}{|l|l|l|}
\hline System & Resources & Description \\
\hline \hline BiBr.EF.1 & Limited & baseline of bilingual bracketing \\
BiBr.EF.2 & Unlimited & baseline of bilingual bracketing + English POS tagging \\
BiBr.EF.3 & Unlimited & baseline of bilingual bracketing + English POS tagging and base NP \\
BiBr.EF.4 & Limited & reverse direction of BiBr.EF.1 \\
BiBr.EF.5 & Unlimited & reverse direction of BiBr.EF.2 \\
BiBr.EF.6 & Unlimited & reverse direction of BiBr.EF.3 \\
BiBr.EF.7 & Limited & intersection of BiBr.EF.1 \& BiBr.EF.3 \\
BiBr.EF.8 & Unlimited & intersection of BiBr.EF.3 \& BiBr.EF.6 \\
\hline \hline ProAlign.EF.1 & Unlimited & cohesion between source and target language + English parser + \\
& & distributional similarity for English words \\
\hline \hline Ralign.EF.1 & Limited & Giza (IBM Model 2) + recursive parallel segmentation \\
\hline \hline UMD.EF.1 & Limited & IBM Model 2, trained with 1/20 of the corpus, distortion 2, iterations 4 \\
\hline \hline XRCE.Base.EF.1 & Limited & GIZA++ (IBM Model 4) with English and French lemmatizer \\
XRCE.Nolem.EF.2 & Limited & GIZA++ only (IBM Model 4), trained with 1/4 of the corpus \\
XRCE.Nolem.EF.3 & Limited & GIZA++ only (IBM Model 4), trained with 1/2 of the corpus \\
\hline
\end{tabular}

Table 2: Short description for English-French systems

\begin{tabular}{|c|c|c|}
\hline System & Resources & Description \\
\hline$\overline{\text { BiBr.RE.1 }}$ & Limited & baseline of bilingual bracketing \\
\hline BiBr.RE.2 & Unlimited & baseline of bilingual bracketing + English POS tagging \\
\hline BiBr.RE.3 & Unlimited & baseline of bilingual bracketing + English POS tagging and base NP \\
\hline Fourday.RE.1 & Limited & nearest neighbor combination of baseline measures \\
\hline ProAlign.RE.1 & "Unlimited & $\begin{array}{l}\text { cohesion between source and target language + English parser + } \\
\text { distributional similarity for English words }\end{array}$ \\
\hline$\overline{\text { RACAI.RE.1 }}$ & "Unlimited & translation equivalence dictionary (Tufiş, 2002) + POS tagging \\
\hline 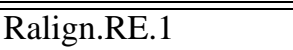 & Limited & "Giza (IBM Model 2) + recursive parallel segmentation \\
\hline UMD.RE.1 & Limited & IBM Model 2, trained with all the corpus, distortion 4 , iterations 4 \\
\hline UMD.RE. 2 & Limited & IBM Model 2, trained with all the corpus, distortion 2, iterations 4 \\
\hline 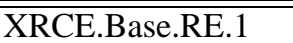 & $\overline{\text { Limited }}$ & GIZA++ (IBM Model 4), with English lemmatizer \\
\hline XRCE.Nolem.RE. 2 & Limited & GIZA++ only (IBM Model 4) \\
\hline XRCE.Trilex.RE.3 & Limited & GIZA++ only (IBM Model 4), with English lemmatizer and trinity le \\
\hline XRCE.Trilex.RE.4 & Limited & GIZA++ only (IBM Model 4), with English lemmatizer and trinity lexicon \\
\hline
\end{tabular}

Table 3: Short description for Romanian-English systems 


\begin{tabular}{|l|c|c|c|c|c|c|c|}
\hline System & $P_{S}$ & $R_{S}$ & $F_{S}$ & $P_{P}$ & $R_{P}$ & $F_{P}$ & AER \\
\hline \multicolumn{7}{|c|}{ Limited Resources } \\
\hline BiBr.RE.1 & $70.65 \%$ & $55.75 \%$ & $62.32 \%$ & $59.60 \%$ & $57.65 \%$ & $58.61 \%$ & $41.39 \%$ \\
Fourday.RE.1 & $0.00 \%$ & $0.00 \%$ & $0.00 \%$ & $52.83 \%$ & $42.86 \%$ & $47.33 \%$ & $52.67 \%$ \\
Ralign.RE.1 & $\mathbf{9 2 . 0 0 \%}$ & $45.06 \%$ & $60.49 \%$ & $63.63 \%$ & $\mathbf{6 5 . 9 2 \%}$ & $64.76 \%$ & $35.24 \%$ \\
UMD.RE.1 & $57.67 \%$ & $49.70 \%$ & $53.39 \%$ & $57.67 \%$ & $49.70 \%$ & $53.39 \%$ & $46.61 \%$ \\
UMD.RE.2 & $58.29 \%$ & $49.99 \%$ & $53.82 \%$ & $58.29 \%$ & $49.99 \%$ & $53.82 \%$ & $46.18 \%$ \\
XRCE.Base.RE.1 & $79.28 \%$ & $61.14 \%$ & $69.03 \%$ & $79.28 \%$ & $61.14 \%$ & $\mathbf{6 9 . 0 3 \%}$ & $30.97 \%$ \\
XRCE.Nolem-56K.RE.2 & $82.65 \%$ & $\mathbf{6 2 . 4 4 \%}$ & $\mathbf{7 1 . 1 4 \%}$ & $\mathbf{8 2 . 6 5 \%}$ & $62.44 \%$ & $71.14 \%$ & $\mathbf{2 8 . 8 6 \%}$ \\
XRCE.Trilex.RE.3 & $80.97 \%$ & $61.89 \%$ & $70.16 \%$ & $80.97 \%$ & $61.89 \%$ & $70.16 \%$ & $29.84 \%$ \\
XRCE.Trilex.RE.4 & $79.76 \%$ & $61.31 \%$ & $69.33 \%$ & $79.76 \%$ & $61.31 \%$ & $69.33 \%$ & $30.67 \%$ \\
\hline \hline \multicolumn{7}{|c|}{ Unlimited Resources } \\
\hline BiBr.RE.2 & $70.46 \%$ & $55.51 \%$ & $62.10 \%$ & $58.40 \%$ & $57.59 \%$ & $57.99 \%$ & $41.39 \%$ \\
BiBr.RE.3 & $70.36 \%$ & $55.47 \%$ & $62.04 \%$ & $58.17 \%$ & $58.12 \%$ & $58.14 \%$ & $41.86 \%$ \\
RACAI.RE.1 & $81.29 \%$ & $\mathbf{6 0 . 2 6 \%}$ & $69.21 \%$ & $81.29 \%$ & $\mathbf{6 0 . 2 6 \%}$ & $69.21 \%$ & $30.79 \%$ \\
ProAlign.RE.1 & $\mathbf{8 8 . 2 2 \%}$ & $58.91 \%$ & $\mathbf{7 0 . 6 4 \%}$ & $\mathbf{8 8 . 2 2 \%}$ & $58.91 \%$ & $\mathbf{7 0 . 6 4 \%}$ & $\mathbf{2 9 . 3 6 \%}$ \\
\hline
\end{tabular}

Table 4: Results for Romanian-English, NO-NULL-Align

\begin{tabular}{|l|c|c|c|c|c|c|c|}
\hline System & $P_{S}$ & $R_{S}$ & $F_{S}$ & $P_{P}$ & $R_{P}$ & $F_{P}$ & AER \\
\hline & Limited Resources \\
\hline BiBr.RE.1 & $70.65 \%$ & $48.32 \%$ & $57.39 \%$ & $57.38 \%$ & $52.62 \%$ & $54.90 \%$ & $45.10 \%$ \\
Fourday.RE.1 & $0.00 \%$ & $0.00 \%$ & $0.00 \%$ & $35.85 \%$ & $45.88 \%$ & $40.25 \%$ & $59.75 \%$ \\
Ralign.RE.1 & $\mathbf{9 2 . 0 0 \%}$ & $39.05 \%$ & $54.83 \%$ & $63.63 \%$ & $57.13 \%$ & $60.21 \%$ & $39.79 \%$ \\
UMD.RE.1 & $56.21 \%$ & $43.17 \%$ & $48.84 \%$ & $45.51 \%$ & $47.76 \%$ & $46.60 \%$ & $53.40 \%$ \\
UMD.RE.2 & $56.58 \%$ & $43.45 \%$ & $49.15 \%$ & $46.00 \%$ & $47.88 \%$ & $46.92 \%$ & $53.08 \%$ \\
XRCE.Base.RE.1 & $79.28 \%$ & $52.98 \%$ & $63.52 \%$ & $61.59 \%$ & $61.50 \%$ & $61.54 \%$ & $38.46 \%$ \\
XRCE.Nolem-56K.RE.2 & $82.65 \%$ & $\mathbf{5 4 . 1 2 \%}$ & $\mathbf{6 5 . 4 1 \%}$ & $61.59 \%$ & $61.50 \%$ & $61.54 \%$ & $38.46 \%$ \\
XRCE.Trilex.RE.3 & $80.97 \%$ & $53.64 \%$ & $64.53 \%$ & $\mathbf{6 3 . 6 4 \%}$ & $\mathbf{6 1 . 5 8 \%}$ & $\mathbf{6 2 . 5 9 \%}$ & $\mathbf{3 7 . 4 1 \%}$ \\
XRCE.Trilex.RE.4 & $79.76 \%$ & $53.14 \%$ & $63.78 \%$ & $62.22 \%$ & $61.37 \%$ & $61.79 \%$ & $38.21 \%$ \\
\hline \hline \multicolumn{8}{|c|}{ Unlimited Resources } \\
\hline BiBr.RE.2 & $70.46 \%$ & $48.11 \%$ & $57.18 \%$ & $56.01 \%$ & $52.26 \%$ & $54.07 \%$ & $45.93 \%$ \\
BiBr.RE.3 & $70.36 \%$ & $48.08 \%$ & $57.12 \%$ & $56.05 \%$ & $52.87 \%$ & $54.42 \%$ & $45.58 \%$ \\
RACAI.RE.1 & $60.30 \%$ & $\mathbf{6 2 . 3 8 \%}$ & $61.32 \%$ & $59.87 \%$ & $\mathbf{6 2 . 4 2 \%}$ & $61.12 \%$ & $38.88 \%$ \\
ProAlign.RE.1 & $\mathbf{8 8 . 2 2 \%}$ & $51.06 \%$ & $\mathbf{6 4 . 6 8 \%}$ & $\mathbf{6 1 . 7 1 \%}$ & $62.05 \%$ & $\mathbf{6 1 . 8 8 \%}$ & $\mathbf{3 8 . 1 2 \%}$ \\
\hline
\end{tabular}

Table 5: Results for Romanian-English, NULL-Align 


\begin{tabular}{|l|c|c|c|c|c|c|c|}
\hline System & $P_{S}$ & $R_{S}$ & $F_{S}$ & $P_{P}$ & $R_{P}$ & $F_{P}$ & AER \\
\hline \multicolumn{8}{|c|}{ Limited Resources } \\
\hline BiBr.EF.1 & $49.85 \%$ & $79.45 \%$ & $61.26 \%$ & $67.23 \%$ & $29.24 \%$ & $40.76 \%$ & $28.23 \%$ \\
BiBr.EF.4 & $51.46 \%$ & $82.42 \%$ & $63.36 \%$ & $66.65 \%$ & $32.68 \%$ & $43.86 \%$ & $28.01 \%$ \\
BiBr.EF.7 & $63.03 \%$ & $74.59 \%$ & $68.32 \%$ & $66.11 \%$ & $30.06 \%$ & $41.33 \%$ & $29.38 \%$ \\
Ralign.EF.1 & $\mathbf{7 2 . 5 4 \%}$ & $80.61 \%$ & $\mathbf{7 6 . 3 6 \%}$ & $77.56 \%$ & $\mathbf{3 8 . 1 9 \%}$ & $\mathbf{5 1 . 1 8 \%}$ & $18.50 \%$ \\
UMD.EF.1 & $37.98 \%$ & $64.66 \%$ & $47.85 \%$ & $59.69 \%$ & $23.53 \%$ & $33.75 \%$ & $38.47 \%$ \\
XRCE.Base.EF.1 & $50.89 \%$ & $84.67 \%$ & $63.57 \%$ & $83.22 \%$ & $32.05 \%$ & $46.28 \%$ & $16.23 \%$ \\
XRCE.Nolem.EF.2 & $55.54 \%$ & $\mathbf{9 3 . 4 6 \%}$ & $69.68 \%$ & $89.65 \%$ & $34.92 \%$ & $50.27 \%$ & $8.93 \%$ \\
XRCE.Nolem.EF.3 & $55.43 \%$ & $93.81 \%$ & $69.68 \%$ & $\mathbf{9 0 . 0 9 \%}$ & $35.30 \%$ & $50.72 \%$ & $\mathbf{8 . 5 3 \%}$ \\
\hline \hline \multicolumn{8}{|c|}{ Unlimited Resources } \\
\hline BiBr.EF.2 & $50.05 \%$ & $79.89 \%$ & $61.54 \%$ & $66.92 \%$ & $29.14 \%$ & $40.60 \%$ & $28.24 \%$ \\
BiBr.EF.3 & $50.21 \%$ & $80.26 \%$ & $61.80 \%$ & $63.79 \%$ & $30.52 \%$ & $41.29 \%$ & $30.38 \%$ \\
BiBr.EF.5 & $51.27 \%$ & $82.17 \%$ & $63.15 \%$ & $67.22 \%$ & $32.56 \%$ & $43.87 \%$ & $27.71 \%$ \\
BiBr.EF.6 & $51.91 \%$ & $83.26 \%$ & $63.95 \%$ & $62.21 \%$ & $\mathbf{3 4 . 5 8 \%}$ & $\mathbf{4 4 . 4 5 \%}$ & $31.32 \%$ \\
BiBr.EF.8 & $66.34 \%$ & $74.86 \%$ & $70.34 \%$ & $61.62 \%$ & $31.37 \%$ & $41.57 \%$ & $32.48 \%$ \\
ProAlign.EF.1 & $\mathbf{7 1 . 9 4 \%}$ & $\mathbf{9 1 . 4 8 \%}$ & $\mathbf{8 0 . 5 4 \%}$ & $\mathbf{9 6 . 4 9 \%}$ & $28.41 \%$ & $43.89 \%$ & $\mathbf{5 . 7 1 \%}$ \\
\hline
\end{tabular}

Table 6: Results for English-French, NO-NULL-Align

\begin{tabular}{|l|c|c|c|c|c|c|c|}
\hline System & $P_{S}$ & $R_{S}$ & $F_{S}$ & $P_{P}$ & $R_{P}$ & $F_{P}$ & AER \\
\hline \multicolumn{8}{|c|}{ Limited Resources } \\
\hline BiBr.EF.1 & $49.85 \%$ & $79.45 \%$ & $61.26 \%$ & $60.32 \%$ & $29.12 \%$ & $39.28 \%$ & $33.37 \%$ \\
BiBr.EF.4 & $51.46 \%$ & $82.42 \%$ & $63.36 \%$ & $61.64 \%$ & $32.41 \%$ & $42.48 \%$ & $31.91 \%$ \\
BiBr.EF.7 & $\mathbf{6 3 . 0 3 \%}$ & $74.59 \%$ & $68.32 \%$ & $51.35 \%$ & $30.45 \%$ & $38.23 \%$ & $40.97 \%$ \\
Ralign.EF.1 & $72.54 \%$ & $80.61 \%$ & $\mathbf{7 6 . 3 6 \%}$ & $\mathbf{7 7 . 5 6 \%}$ & $\mathbf{3 6 . 7 9 \%}$ & $\mathbf{4 9 . 9 1 \%}$ & $\mathbf{1 8 . 5 0 \%}$ \\
UMD.EF.1 & $37.19 \%$ & $64.66 \%$ & $47.22 \%$ & $41.93 \%$ & $24.08 \%$ & $30.59 \%$ & $51.71 \%$ \\
XRCE.Base.EF.1 & $50.89 \%$ & $84.67 \%$ & $63.57 \%$ & $64.96 \%$ & $32.73 \%$ & $43.53 \%$ & $28.99 \%$ \\
XRCE.Nolem.EF.2 & $55.54 \%$ & $93.46 \%$ & $69.68 \%$ & $70.98 \%$ & $35.61 \%$ & $47.43 \%$ & $22.10 \%$ \\
XRCE.Nolem.EF.3 & $55.43 \%$ & $\mathbf{9 3 . 8 1 \%}$ & $69.68 \%$ & $72.01 \%$ & $36.00 \%$ & $48.00 \%$ & $21.27 \%$ \\
\hline \hline \multicolumn{8}{|c|}{ Unlimited Resources } \\
\hline BiBr.EF.2 & $50.05 \%$ & $79.89 \%$ & $61.54 \%$ & $59.89 \%$ & $28.96 \%$ & $39.04 \%$ & $33.48 \%$ \\
BiBr.EF.3 & $50.21 \%$ & $80.26 \%$ & $61.80 \%$ & $57.85 \%$ & $30.28 \%$ & $39.75 \%$ & $35.03 \%$ \\
BiBr.EF.5 & $51.27 \%$ & $82.17 \%$ & $63.15 \%$ & $\mathbf{6 2 . 0 5 \%}$ & $32.23 \%$ & $42.43 \%$ & $\mathbf{3 1 . 6 9 \%}$ \\
BiBr.EF.6 & $51.91 \%$ & $83.26 \%$ & $63.95 \%$ & $58.41 \%$ & $\mathbf{3 4 . 2 0 \%}$ & $\mathbf{4 3 . 1 4 \%}$ & $34.47 \%$ \\
BiBr.EF.8 & $66.34 \%$ & $74.86 \%$ & $70.34 \%$ & $48.50 \%$ & $31.76 \%$ & $38.38 \%$ & $43.37 \%$ \\
ProAlign.EF.1 & $\mathbf{7 1 . 9 4 \%}$ & $\mathbf{9 1 . 4 8 \%}$ & $\mathbf{8 0 . 5 4 \%}$ & $56.02 \%$ & $30.05 \%$ & $39.62 \%$ & $33.71 \%$ \\
\hline
\end{tabular}

Table 7: Results for English-French, NULL-Align 


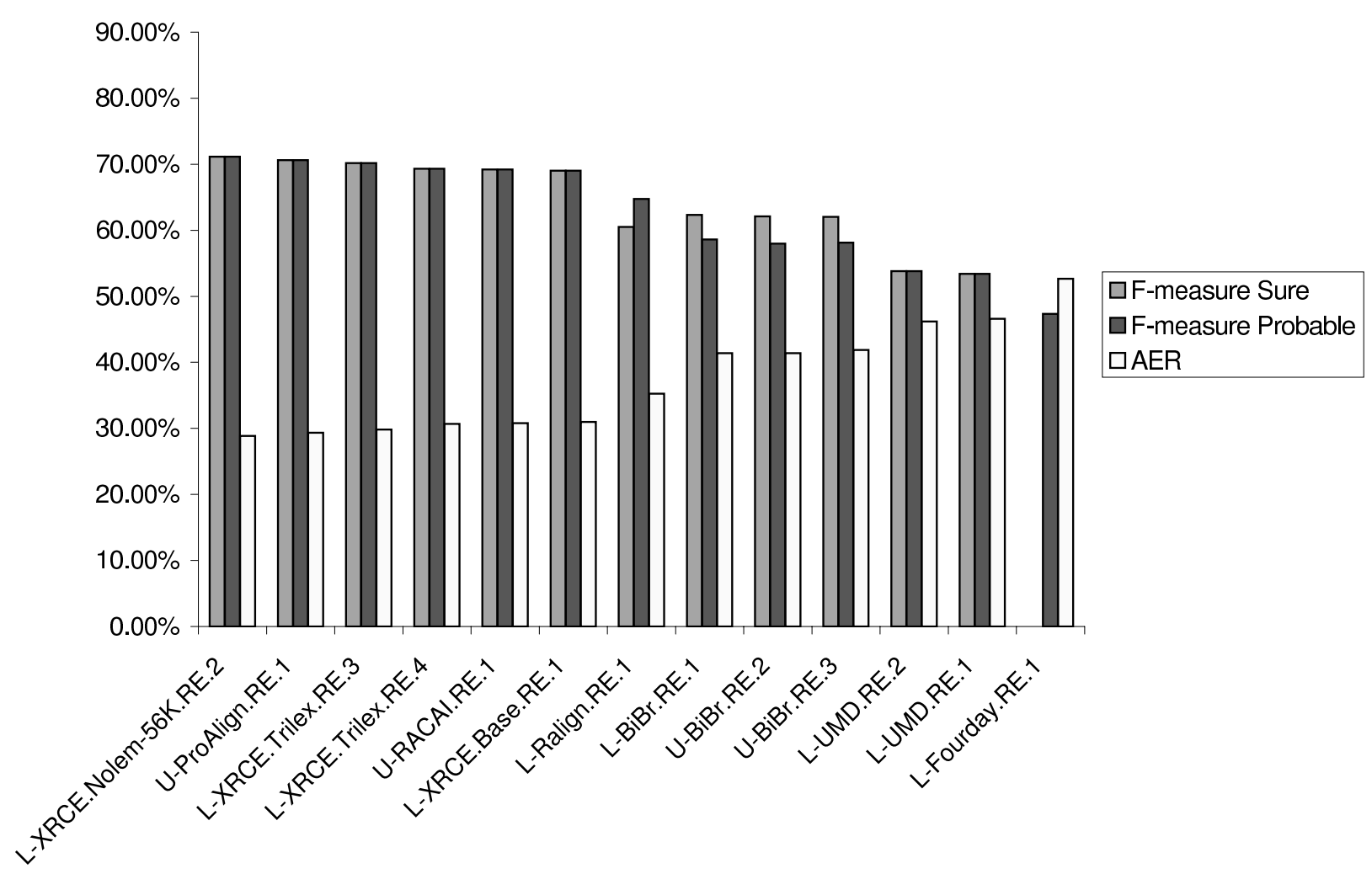

Figure 2: Ranked results for Romanian-English data

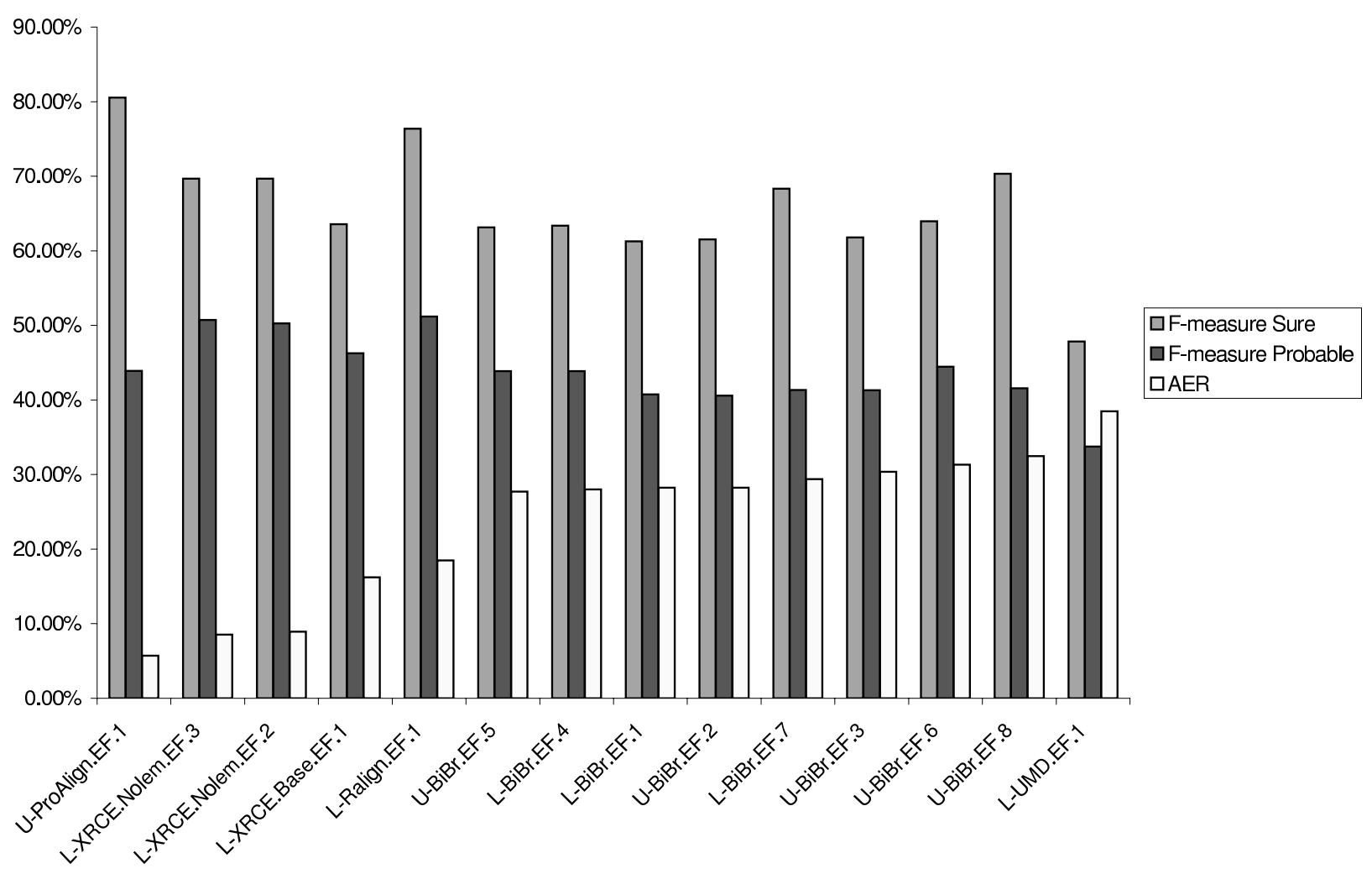

Figure 3: Ranked results for English-French data 